\title{
Deep Learning for Multi Grade Brain Tumor Classification in Smart Healthcare Systems: A Prospective Survey
}

\author{
Sravya V J ${ }^{\mathrm{a}, 1}$ Malathi $\mathrm{S}^{\mathrm{b}}$ \\ ${ }^{a} P G$ Scholar, Dept of CSE, Panimalar Engineering College, Chennai, India \\ ${ }^{\mathrm{b}}$ Professor, Dept of CSE, Panimalar Engineering College, Chennai, India
}

\begin{abstract}
Cancer is one the dangerous disease for human mankind. There are different types of cancers and the dangerous among them is Brain tumor cancer. Though radilogists are able to diagnose the brain tumor, they find difficulties in identifying grade of brain tumor. In the digitally advanced field, the grade identification of brain tumor still seems a challenging task. There are multiple survey that study the identification of brain tumor. But identifying grade of brain tumor is one area which needs more focus. State of the art convolution network is an key algorithm to identify brain tumor grades. We explore convolution network with transfer learning techniques and data augmentation to achieve more accuracy in detecting appropriate grade of brain tumor. We also detail describe various techniques used prior to our study and how our study enhances from currentmechanism.
\end{abstract}

Keywords - multi grade, brain tumour, deep learning, health monitoring, image processing, intelligent healthcare

\section{Introduction}

Brain tumor has gained lot of researcher's attention with advancement in technology in the field of medical image processing. Death rate due to brain tumor keeps on increasing year by year, stated by Nation Brain Tumor Foundation. Identifying brain tumor and predicting the type is major challenge. Medical images are examined by experts and for identifying brain tumor. Nature of the image adds up complexity for diagnosing with human eye because accurate detection and precise location of cancer cells are required for critical diagnosis. Expert diagnosis is very much required for accurate segmentation and classification. Complete manual diagnosis is time consuming and also fully automated solutions are yet to be achieved. These automated system demand second opinion as we deal with life. While we mostly depend on expert advice, several studies have proposed automated solution or semi-automated solution based on historical data in the absence of experts. World Health Organization(WHO) has studied different types of brain tumor and provided the classification standards for brain tumor. They have classified the brain tumor from Grade I to Grade IV wherein

\footnotetext{
${ }^{1}$ Sravya.V, PG Scholar, Dept of CSE, Panimalar Engineering College, Chennai, India;

E-mail:sravya.krupakar@gmail.com
} 
Grade I is considered as benign and Grade IV is considered as highly malign. Glioblastomos, the grade IV is classified as most dangerous type of brain tumour and Histopathology is the most preferred method to identify grade IV tumours from other grades of brain tumour. Different features of grade IV brain tumours are necrosis, vascular thrombosis, vascular proliferation. These features are used to differntiate grade IV brain tumour from others. These features are not transparent and difficult to recognize. The difficulties in identifying these features in native eyes leads to different opinion among pathologists. Histopathological methodology has defects and this results in gliomas unclear. MRI is widely used to overcome the challenges in histopathological methodology. The major advantage here is MRI is able to detect diverse tissues. Also with MRI, varied tissue outline configuration is achieved. Also with appropriate sequence, MRI technology was able to successfully seperate low grade tumors from grade IV malignant tumour.

\section{Related work}

We have collected all the research dataset on brain tumour along with their benchmarks. we have focussed on survey of brain tumour classification and its predominant diagnosis techniques, advantages and limitations

1. Deep learning has evolved a lot and found major industry break through in the field of image processing. We analyzed transfer learning techniques from deep learning models for brain tumor with and without augmentation of data. Statistics of these models with various parameters are detailed out in our study.

2. With smart device being turning the table with evolution of the decade, we have focused the study on usage of smart devices and cloud computing. Solutions integrated with these technologies will be the future.

3. Different deep learning methodologies are studied to spot the major challenge in the domain of Brain tumor classification under healthcare. Recommendation and future study of different methodologies were also focused

Table 1. Method adopted so far and their accuracy and drawbacks which paved the way for the emergence of new methodology

\begin{tabular}{cccc}
\hline S: NO & METHODOLOGY ACCURACY & DRAWBACKS \\
\hline 1. & Noise Removal & $95 \%$ & $\begin{array}{c}\text { They degrade image details and edges of } \\
\text { the image. Therefore noised } \\
\text { image would be blurred }\end{array}$ \\
2. & Image Processing & $80 \%$ & Image takes gets ruptured as days goes by \\
3. & Preprocessing & $30 \%$ & $\begin{array}{c}\text { Have some unwanted distortions or } \\
\text { enhances some image features important } \\
\text { for further processing. }\end{array}$ \\
\end{tabular}




\begin{tabular}{|c|c|c|c|}
\hline 4. & segmentation & $25 \%$ & $\begin{array}{l}\text { The least method used as they have } \\
\text { various error in working. }\end{array}$ \\
\hline 5. & Feature extraction & $67 \%$ & Image dets destroyed or get faded. \\
\hline 6. & classification & $70 \%$ & $\begin{array}{l}\text { The disadvantage is that the accuracy } \\
\text { depends on the classification accuracy }\end{array}$ \\
\hline 7. & $\mathrm{CNN}$ & $87 \%$ & $\begin{array}{l}\text { lack of GPU in system make CNN trainin£ } \\
\text { time high for training, if it has more layers }\end{array}$ \\
\hline 8. & Training & $90 \%$ & $\begin{array}{c}\text { Training majorly depends on quality of } \\
\text { data. }\end{array}$ \\
\hline 9. & Gray scaling & $86 \%$ & $\begin{array}{l}\text { Slicing out to get perfect image leads to } \\
\text { data loss. }\end{array}$ \\
\hline 10. & AlexNet & $75 \%$ & Possibilities of malfunction on running. \\
\hline 11. & GoogleNet & $89 \%$ & $\begin{array}{c}\text { Google Net proposed architecture promise } \\
\text { on better performance }\end{array}$ \\
\hline 12. & Neural Network & $60 \%$ & Local minima. Over-fitting \\
\hline 13. & Machine learning & $40 \%$ & Makes the system more complex. \\
\hline 14. & KNN & $30 \%$ & Sensitive to noise. \\
\hline 15. & SVM & $54 \%$ & $\begin{array}{l}\text { Testing is slow } \\
\text { No desired output is obtained. }\end{array}$ \\
\hline 16. & MobileNet & $70 \%$ & $\begin{array}{l}\text { It is less accurate than other state-of-the- } \\
\text { art networks }\end{array}$ \\
\hline 17. & Ensemble learning & $30 \%$ & Does not provide the required result \\
\hline 18. & $\begin{array}{l}\text { Probabilistic } \\
\text { neural network }\end{array}$ & $15 \%$ & Complexity of the system is high. \\
\hline 19. & Decision tree & $45 \%$ & Unstable classifier. Performance low \\
\hline 20. & Behavior analysis & $40 \%$ & Not predictable \\
\hline
\end{tabular}

\subsection{Methodology}

We have used clinical dataset of brain tumor for our project. The dataset contains MRI images of benign and malign brain tumors. The raw images needs to be pre-processed. Later augmentation and segmentation techniques need to be applied on the preprocessed data. Deep Learning techniques will be applied after splitting the data into 
training, validation and testing data. CNN alogrthim is optmized with appropriate hyperparamter tuning. Majority of the data contains noise the the form of patients data in text format. The RGB data is converted into gray scale by applying weighted average technique. In medical field, images are mostly affected by salt and pepper noise. To suppress the noise by salt and pepper, the effective method of median filter is applied. We apply median filter at very early stage of project lifecycle to remove the noise and improve accuracy. Segmentation is a key area for brain tumor analysis. This effective extracts the region of brain from the skull. Thresholding methodology is used to get the piece of brain from the skull as part of segmentation. Morphological methods such as dilation and erosion to get the appropriate shape. Since deep learning techniques does not work effectively when data is less, we apply data augmentation technique to increase the size of the data. This also helps to avoid overfitting of data. Deep learning is widely used for classification after major break through. CNN is the mostly used among Deep learning for image processing. Considering the accuracy of $\mathrm{CNN}$, and its usage across image processing, we have used $\mathrm{CNN}$ for our multi grade brain tumor classification problem. CNN learns the spatial correlation between pixels better by studying them in hierarchical method. This is achieved with convolving image with feature maps. Later size of the features is reduced with max pooling layer and lately falttened by applying feed to dense layer.

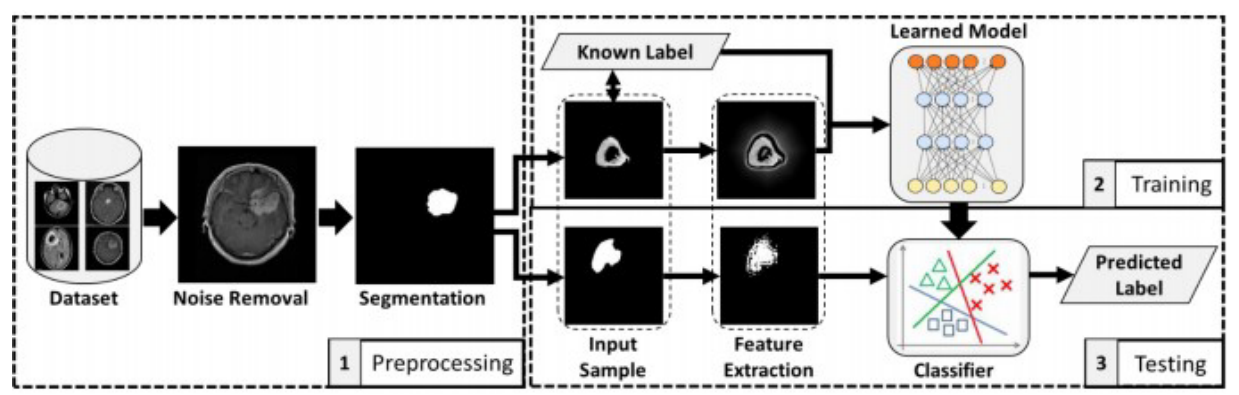

Figure 1. Architectural diagram

\section{Conclusion and future work}

Considering the recent development in the domain of BTC and the limitations of existing studies, we presented a comprehensive survey of deep learning-based BTC methods. Deep learning technologies accurately assist radiologists in predicting the tumor regions and further classifying them into their respective types. Many researchers contributed to the field of BTC, but many challenges remain therein. Therefore, we conducted this study to provide the overall literature of deep learningbased BTC methods in a single survey and to draw the attention of both academia and industry toward the necessary development in this domain. This article comprehensively discussed all deep learning-based BTC methods, with their achievements and weaknesses, followed by complete information about the existing publicly available data sets with their respective resources. In order to empirically inform the conclusions drawn from our literature study, we experimentally analyze various deep learning models by performing extensive experiments over BTC data sets and highlighted the suitable option for consideration in smart health care. Finally, this study highlighted key 
challenges, such as lack of public data sets and end-to-end deep learning models, and suggested detailed directions for further research in BTC domain, i.e., exploring edge/fog/cloud computing with FA, advanced data-enrichment techniques, model confidence and explainability, IoMT, and deep investigation of sequential and transfer learning strategies. This can increase the maturity level of BTC methods with better applicability for commercial clinical applications and their smooth integration with smart healthcare. The brain is an intriguing system whose complexity demands sophisticated means to understand and characterize its behavior. The unrivaled learning capability of deep learning models has made them the standard choice to detect and classify brain tumors from MRI images and other monitored data alike, spawning a flurry of research activity overviewed in this survey. We hope that the numerous research paths outlined in our overview will serve as supportive material for the research community currently working on this field and a stimulating read for newcomers to this domain.

\section{References}

[1] C.-M. Feng, Y. Xu, J.-X. Liu, Y.-L. Gao, and C.-H. Zheng, Supervised discriminative sparse PCA for com-characteristic gene selection and tumor classification on multiview biological data, IEEE Trans. Neural Netw. Learn. Syst., vol. 30, no. 10, pp. 2926-2937, Oct. 2019.

[2] A. Ruiz-Garcia, N. Webb, V. Palade, M. Eastwood, and M. Elshaw, Deep learning for real time facial expression recognition in social robots, in Proc. Int. Conf. Neural Inf. Process., 2018, pp. 392402.

[3] J. Sun, C. Li, X.-J. Wu, V. Palade, and W. Fang, An effective method of weld defect detection and classification based on machine vision, IEEE Trans. Ind. Informat., vol. 15, no. 12, pp. 6322-6333, Dec. 2019.

[4] M. Mahmud, M. S. Kaiser, A. Hussain, and S. Vassanelli, Applications of deep learning and reinforcement learning to biological data, IEEE Trans. Neural Netw. Learn. Syst., vol. 29, no. 6, pp. 2063-2079, Jun. 2018.

[5] P. Chriskos, C. A. Frantzidis, P. T. Gkivogkli, P. D. Bamidis, and C. Kourtidou- Papadeli, Automatic sleep staging employing convolutional neural networks and cortical connectivity images, IEEE Trans. Neural Netw. Learn. Syst., vol. 31, no. 1, pp. 113-123, Jan. 2020.

[6] Y. Shi, H.-I. Suk, Y. Gao, S.-W. Lee, and D. Shen, Leveraging coupled interaction for multimodal Alzheimer's disease diagnosis, IEEE Trans. Neural Netw. Learn. Syst., vol. 31, no. 1, pp. 186-200, Jan. 2020.

[7] I. Almakky, V. Palade, Y.-L. Hedley, and J. Yang, A stacked deep autoencoder model for biomedical figure classification, in Proc. IEEE 15th Int. Symp. Biomed. Imag. (ISBI), Apr. 2018, pp. 1134-1138.

[8] Ambeth Kumar.V.D Dr.S.Malathi, V.D.Ashok Kumar (2015).Performance Improvement Using an Automation System for Segmentation of Multiple Parametric Features Based on Human Footprint. for the Journal of Electrical Engineering \& Technology (JEET), vol. 10, no. 4, pp.1815-1821 , 2015. [http://dx.doi.org/10.5370/JEET.2015.10.4.1815]

[9] Ambeth Kumar.V.D et.al .A Survey on Face Recognition in Video Surveillance. Lecturer Notes on Computational and Mechanism, Vol. 30, pp: 699-708, 2019

[10] Ambeth Kumar.V.D .Precautionary measures for accidents due to mobile phone using IOT. Clinical eHealth, Volume 1, Issue 1, March 2018, Pages 30-35.

[11] S.V. Ruphitha et.al, " Management of Major Postpartum Haemorrhage by using Zigbee protocol - A Review “, 2021 6th International Conference on Inventive Computation Technologies (ICICT) (DOI: 10.1109/ICICT50816.2021.9358757 )

[12] M. Indhumathi et.al , "Healthcare Management of Major Cardiovascular Disease-A review", 2021 6th International Conference on Inventive Computation Technologies (ICICT), (DOI: 10.1109/ICICT50816.2021.9358519) 